\title{
Retrospective analysis of the application of CT scan in the emergency department to screen clinically asymptomatic COVID-19 before hospital admission
}

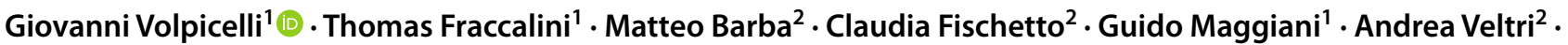 \\ Luciano Cardinale ${ }^{2}$
}

Received: 20 November 2021 / Accepted: 3 January 2022 / Published online: 8 January 2022

(c) American Society of Emergency Radiology 2022

\begin{abstract}
Background The necessity to identify and isolate COVID-19 patients to avoid intrahospital cross infections is particularly felt as a challenge. Clinically occult SARS-CoV-2 infection among patients admitted to the hospital is always considered a risk during the pandemic. The aim of our study is to describe the application of CT scan to reveal unexpected COVID-19 in patients needing hospital admission.

Method In our emergency department, we prospectively enrolled adult patients needing hospital admission, without symptoms suspected of COVID-19, and showing negative reverse transcriptase-polymerase chain reaction (RT-PCR) swab test. CT scan was performed to diagnose clinically occult COVID-19 pneumonia. All the exams were read and discussed retrospectively by two expert radiologists and assigned to one of 4 exclusive diagnoses: typical (typCT), indeterminate (indCT), atypical (atyCT), negative (negCT). The clinical characteristics and final diagnoses were described and compared with the results of CT scans.

Results From May 25 to August 18, 2020, we prospectively enrolled 197 patients. They showed 122 negCT, 52 atyCT, 22 indCT, and 1 typCT. Based on the CT imaging, the prevalence of suspected clinically occult COVID-19 pneumonia was $11.6 \%$ (23 patients). None had confirmation of SARS-CoV-2 infection after the hospital stay. Nineteen patients had negative serial RT-PCR while in 4 cases, the infection was excluded by clinical follow-up or appearance of positivity of RT-PCR after months.

Conclusion Our descriptive analysis confirms that CT scan cannot be considered a valid tool to screen clinically occult COVID-19, when the asymptomatic patients need hospitalization for other conditions. Application of personnel protections and distancing among patients remains the best strategies to limit the possibility of intrahospital cross-infections.
\end{abstract}

Keywords Thoracic imaging · Chest imaging · Thoracic CT scan · COVID-19 pandemic

\section{Introduction}

The bursting of COVID-19 pandemic is challenging the health systems around the world, both in scarce-resource areas and even in the most advanced and rich countries. One of the major challenges posed by the pandemic is

Giovanni Volpicelli

giovi.volpicelli@gmail.com

1 Department of Emergency Medicine, San Luigi Gonzaga University Hospital, Orbassano, Turin, Italy

2 Department of Oncology, Radiology Unit, San Luigi Gonzaga University Hospital, Orbassano, Turin, Italy the management of the surge of admissions, at the same time isolating infected patients to limit in-hospital crossinfections. The first diagnosis of SARS-CoV-2 infection in suspected patients is based on the confirmation by reverse transcriptase-polymerase chain reaction (RT-PCR) nasopharyngeal swab test and visualization of pneumonia by chest imaging, including lung ultrasound and CT scan [1-3]. From the beginning of the first pandemic surge characterized by an extremely high prevalence of infections, it became evident that the sensitivity of the RTPCR was not sufficient to avoid a significant number of false negative cases [4]. In patients with clear symptoms raising suspicion, the process for the first diagnosis of COVID-19 is improved by a systematic combination of 
clinical assessment, bedside lung ultrasound or CT scan, and RT-PCR [3]. In some cases, only serial repetitions of the RT-PCR swab during the hospital stay may reveal initial false negative results. However, it is largely demonstrated that SARS-CoV-2 may give asymptomatic infection in a significant percentage of cases. Thus, in patients admitted to the hospital who do not present with symptoms suspected of SARS-CoV-2 infection, there is the possibility of asymptomatic infection. For this reason, during the pandemic, there is the necessity to exclude asymptomatic SARS-CoV-2 in patients admitted to the hospital for other pathologies, to isolate unexpected new cases and limit the danger of cross-infections. In the recent literature, it was demonstrated that CT scan imaging may be useful to reveal signs of the typical SARS-CoV-2 pneumonia in patients without symptoms and negative RT-PCR [5]. To limit the eventuality of mixing in the same areas of the hospital infected and non-infected patients during a COVID-19 pandemic surge, it is crucial to standardize a strategy that may go from strengthening the procedures for distancing and using of personal protective equipment for patients and personnel, to the more complex and expensive separation of infected and free areas.

The purpose of our study was to evaluate the usefulness of a diagnostic strategy that aims to identify clinically occult COVID-19 pneumonia by thoracic CT scanning performed in patients admitted to the hospital without symptoms of SARS-CoV-2 infection.

\section{Material and methods}

\section{Epidemiological and temporal framework}

From 25 May 2020 to 18 August 2020, we enrolled patients admitted to the San Luigi Gonzaga Hospital in Torino, Italy. Our institution is a university hospital serving the population of Torino, the main town of the Piemonte Region in the north-west of Italy, and neighboring small towns on the west side. The emergency department (ED) of the hospital counts around 45,000 visits per year. During spring 2020, our Region was hit hard by the COVID-19 outbreak with a peak of around 3500 patients admitted to the hospitals in the end of March. After the mid of May in our area, we assisted to a progressive decrease in the prevalence of COVID-19 cases. During the period of enrollment, the prevalence of the disease in our hospital dropped to very few cases still admitted, following the general trend observed in Piemonte (few tens of cases during August). The local ethical committee approved the protocol of this retrospective study (no. 6386 23/4/21).

\section{Selection of the population}

Inclusion criteria were as follows: (1) consecutive patients presenting to the ED and needing admission to the hospital for any medical, surgical, and trauma condition; (2) negative first RT-PCR swab test performed in the ED; (3) absence of symptoms and any other clinical condition raising suspicion of COVID-19; (4) acquisition of a thoracic CT scan before definitive admission, for the diagnosis of clinically occult COVID-19 pneumonia, whose indication was independent from our analysis and based on an internal diagnostic algorithm specifically designed to limit cross-infections inside the hospital (Fig. 1) [6]. Patients of age $>50$ who signed the informed consent received the exam.

\section{RT-PCR swab test}

The diagnosis of COVID-19 was confirmed by a RTPCR nasal-pharyngeal and/or bronchial swab (BD SARS-CoV-2 Reagents for BD MAX System ${ }^{\mathrm{TM}}$ ). In pretriage, a hand-reading Rapid Antigenic Test (COVID19 Ag RAPID TEST DEVICE, Abbott Panbio ${ }^{\mathrm{TM}}$ ) or a facilitated reading (LumiraDx SARS-CoV-2 Ag test LumiraDx) was performed to guide the first allocation of the patients in different areas of the ED. However, the infection was confirmed only after the RT-PCR swab detailed above.

\section{CT scan protocol}

Patients with indication to CT scan signing the informed consent were moved to the radiology unit inside the ED to perform the exam. A multidetector CT (GE OPTIMA 660 General Electric Healthcare, Milwaukee WI, USA) was used. The patients were in supine and head-first position and received scanning with breath held. Parameters used were the following: $100 \mathrm{kV} ; 100$, mAs real-time adaptive control; layer thickness $1-2.5 \mathrm{~mm}$; pitch, 1-1.5; matrix, $512 \times 512$. No contrast was administered. All images were transmitted to the post-processing workstation and reconstructed using high-resolution and conventional algorithms. Each study was read and interpreted by two experts with a long-standing experience in chest imaging. Signs and nomenclature of CT scan were those recommended for COVID-19 and reported in the Radiological Society of North America Expert Consensus document [7]. Each CT scan was assigned to one of the 4 imaging classification recommended in the consensus document: 


\begin{tabular}{|c|c|c|}
\hline \multirow{2}{*}{2} & A.O.U. San Luigi Gonzaga di Orbassano & \\
\cline { 2 - 3 } & Cov_P002.01 & \\
\cline { 2 - 3 } & & Deciou/2020 \\
\hline
\end{tabular}

Patients Allocation in the ED

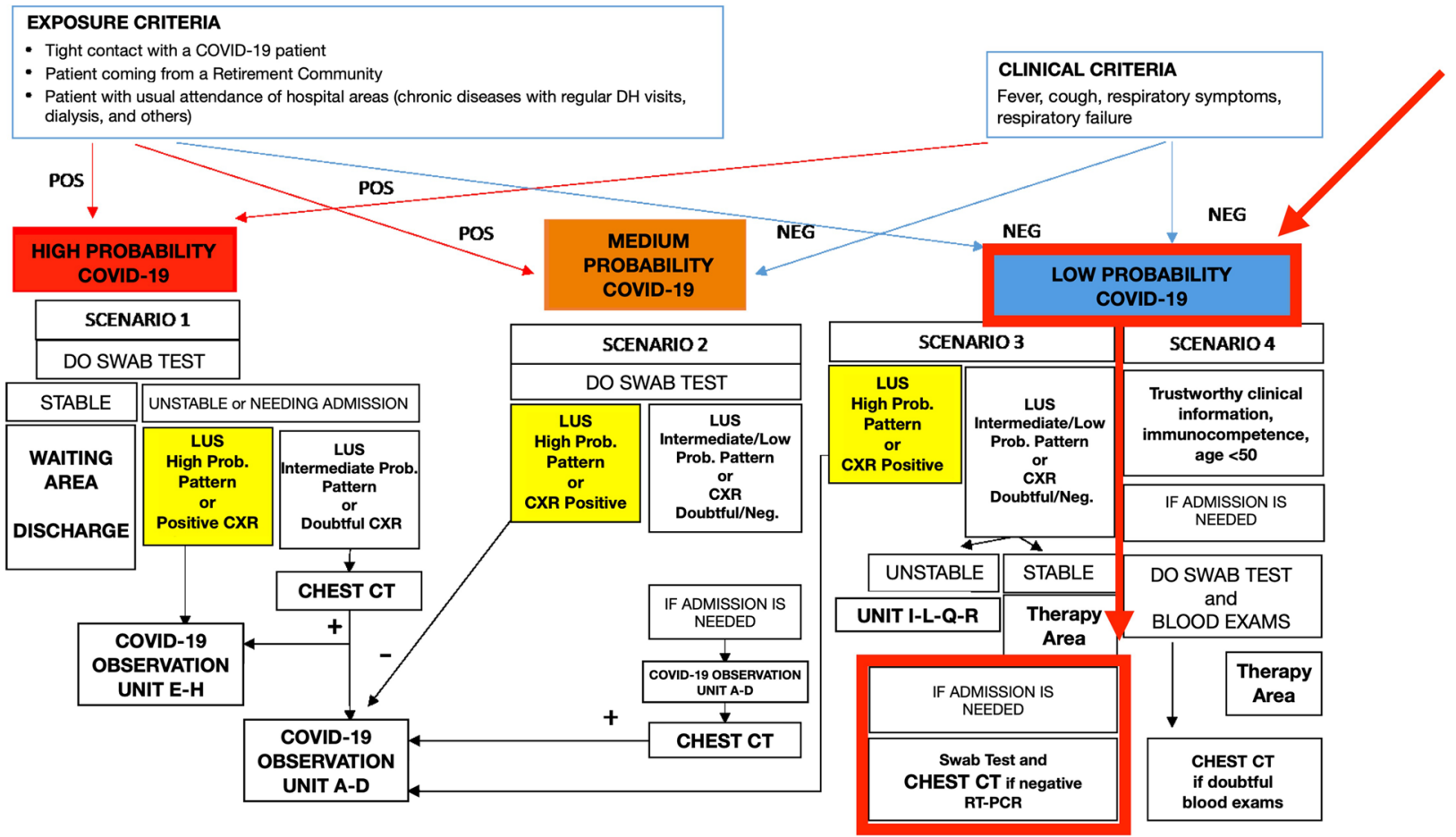

Fig. 1 The original algorithm that was implemented in our institution to guide allocation of patients needing admission, agreed by an internal committee headed by the Director of the Department and the Chiefs of Oncology, Internal Medicine, Pulmonology, Radiology, Emergency Medicine units. The thick red arrows indicate the path dedicated to patients at low probability for COVID-19 and negative RT-PCR, needing admission. After signing an informed consent,

- Typical appearance, in the presence of peripheral, bilateral, multifocal ground glass opacities (GGO) with or without consolidation, or visible lines ("crazy-paving").

- Indeterminate appearance, in the presence of multifocal perihilar or unilateral GGO with or without consolidation, or very small GGO non-rounded or non-peripheral.

- Atypical appearance, in the presence of isolated lobar or segmental consolidation without GGO, or discrete small nodules, or lung cavitation, or smooth interlobular septal thickening with large pleural effusion.

- Negative, in case of no CT features suggesting pneumonia.

\section{Clinical diagnoses}

We focused our analysis on patients with any sign suspected of COVID-19 pneumonia at CT imaging obtained before these patients received CT scan before admission to diagnose unexpected COVID-19 pneumonia. COVID-19, coronavirus disease 2019; DH, day hospital; POS, positive; NEG, negative; Prob., probability; LUS, lung ultrasound; CXR, chest radiography; CT, computed tomography; RT-PCR, reverse transcriptase-polymerase chain reaction

admission (Typical and Indeterminate appearances). To decide about the final diagnoses, we considered the results of repeated RT-PCR swab tests, both nasal-pharyngeal and/or bronchial, and the whole documentation obtained during the hospital stay, as well as, in some doubtful cases, the results of long-term follow-up and monitoring of RT-PCR testing.

\section{Results}

During the period of the study, there were 7900 accesses to the ED with 1336 admissions, of which only 6 patients with a confirmed main diagnosis of COVID-19 pneumonia with respiratory failure. Out of 1330 patients admitted without confirmation of COVID-19 pneumonia, 197 patients responded to the criteria for screening asymptomatic COVID-19 and received a CT scan study before admission from the ED. The calculated total cost of CT 
scans was 24.448 euros net of the time spent by the personnel and cost of supplies. The total amount of irradiation per patient was based on a specific protocol at low dose, giving an average computed tomography dose index volume (CTDIvol) $<5 \mathrm{mGy}$ and dose length product (DLP) $<180 \mathrm{mGy} \times \mathrm{cm}$ per patient. Out of $197 \mathrm{CT}$ studies,
88.3\% ( $n=174$ ) was read Negative or Atypical thus excluding asymptomatic COVID-19 active pneumonia at admission. In $11.7 \%(n=23)$ cases, CT scan was assigned to Typical or Indeterminate appearance (22 Indeterminate and 1 Typical). Figure 2 illustrates the patient's flow of the study and Figs. 3 and 4 show two demonstrative cases with
Fig. 2 The patient's flow diagram showing the flow of participants to our study. COVID19, coronavirus disease 2019; CT, computed tomography

Fig. 3 CT scan of one patient without symptoms of COVID19 and negative RT-PCR swab test. The scan shows bilateral ground glass areas (red arrows) and was interpreted as Typical for COVID-19 interstitial pneumonia, thus raising suspicion of asymptomatic SARS-CoV-2 infection. The patient was admitted into an area of the hospital dedicated to COVID19 , even if in a protected bed, with a main diagnosis of acute myeloid leukemia. During the hospital stay COVID-19 was excluded because of repeated negative RT-PCR tests
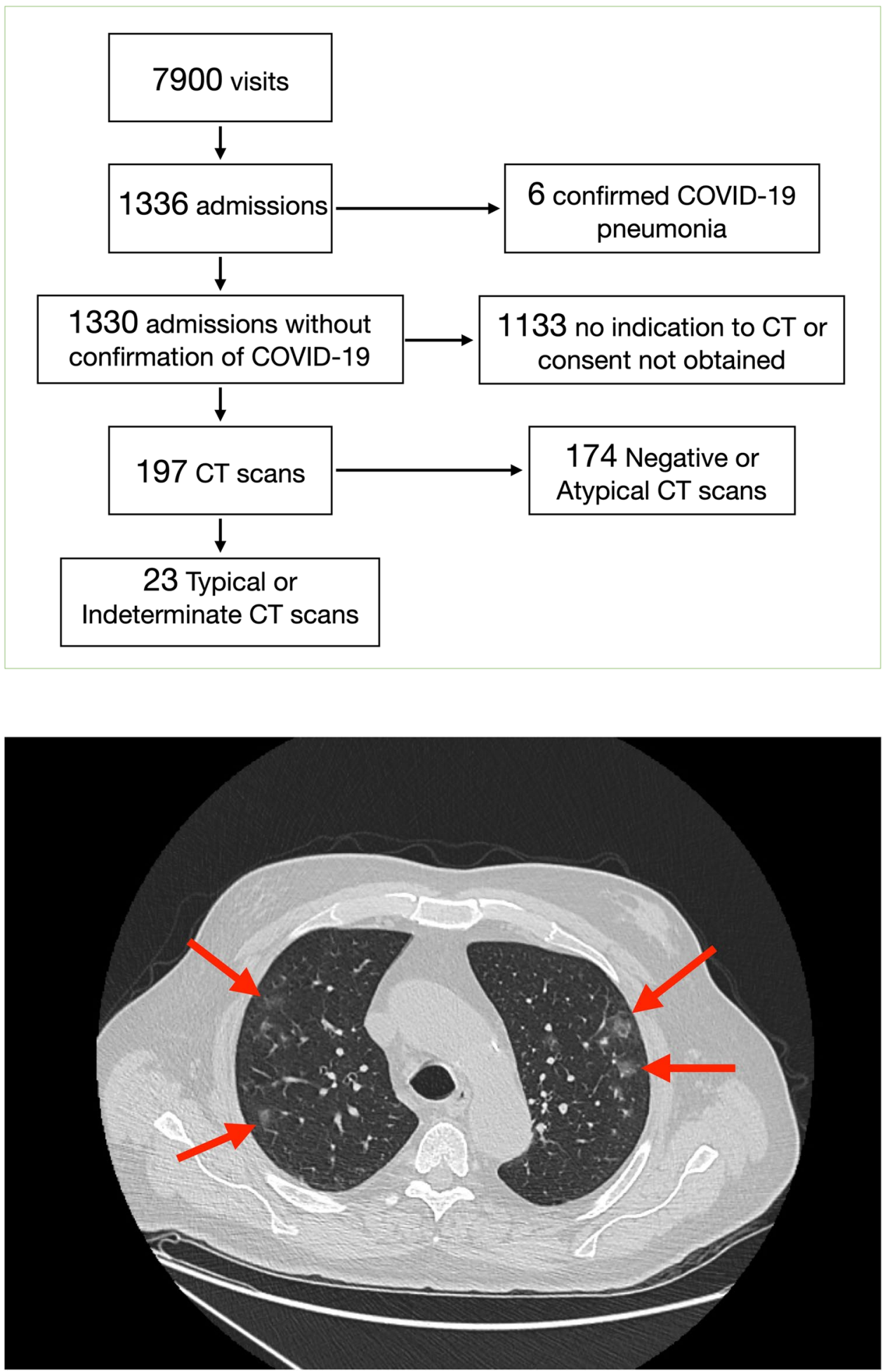


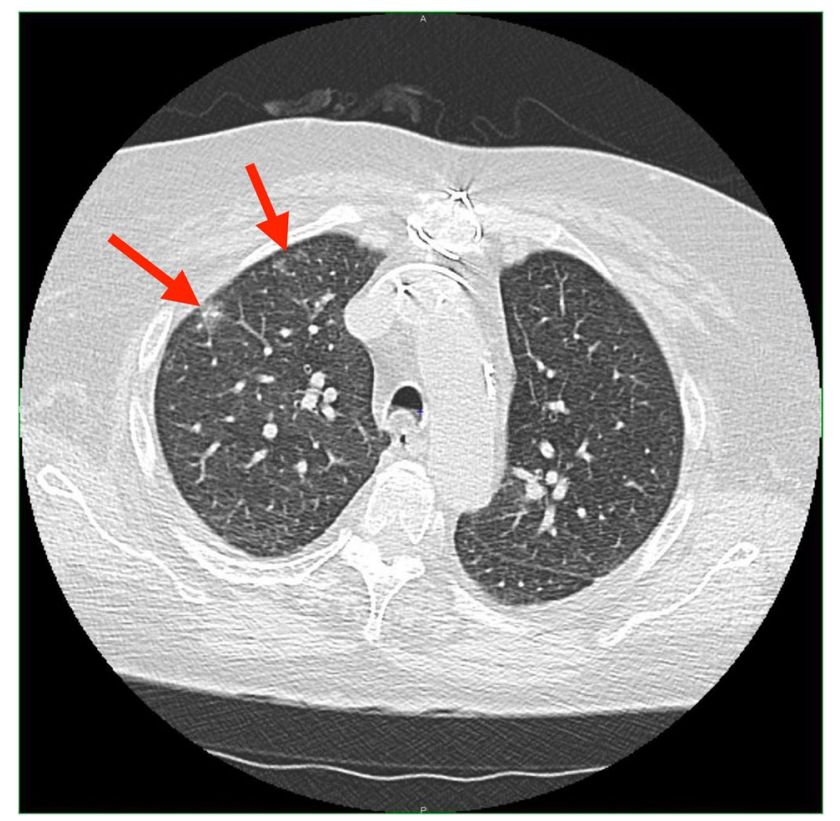

Fig. 4 CT scan of one patient without symptoms of COVID-19 and negative RT-PCR swab test. The scan shows mono-lateral ground glass areas (red arrows) and was interpreted as Indeterminate for COVID-19 interstitial pneumonia, thus raising suspicion of asymptomatic SARS-CoV-2 infection. The patient was admitted into a gray area of the hospital dedicated to suspected COVID-19, with a main diagnosis of bacterial pneumonia. During the hospital stay the RTPCR resulted repeatedly negative and the patient was again admitted to the hospital after some months for COVID-19 pneumonia, this time with positive RT-PCR

Typical and Indeterminate CT readings. The documentations of the 23 cases with suspected COVID-19 pneumonia have been carefully reviewed after the hospital stay to reach a final diagnosis. In Table 1, we report the correspondence between the CT readings, the motivations supporting the status concerning COVID-19 infection at admission and discharge, and the final clinical diagnoses. No patient resulted affected by SARS-CoV-2 infection. In 19 cases, the RT-PCR repeated serially during the hospital stay confirmed the first negativity obtained result in the ED. In 3 cases, the negativity to COVID-19 was also corroborated by the appearance of positivity of the RT-PCR with a new hospital admission after months from the enrollment. In one case, the patient was discharged quickly without repetition of swab tests, but COVID-19 was then excluded because the patient became positive to SARS-CoV-2 and admitted for related pneumonia after months. In 2 cases, a first pulmonary diagnosis of microcitoma and cancer metastases allowed to attribute the CT signs to the neoplastic disease, supported also by the clinical evolution that followed the admissions. In one case of ischemic stroke, the repetition of swab tests was not performed, but the diagnosis of COVID-19 was excluded after clinical follow-up and repetition of chest imaging. In 16 cases out of 23, the patients were admitted to COVID areas or to protected beds (grey zone) inside the COVID areas, with a complementary diagnosis of active infection based on the CT result. In 7 cases, the physician in charge decided to admit the patient in a non-COVID area despite the result of CT. In 21 cases, the diagnosis of COVID-19 at discharge was not confirmed. Only 2 out of 23 patients were still discharged with a probable diagnosis of COVID-19 infection: there was one patient with third-degree atrio-ventricular block who was discharged without the time to perform the RT-PCR surveillance but resulted positive to COVID-19 pneumonia with new hospital admission after months, and one patient who died of septic shock and showed 2 negative RT-PCR tests.

\section{Discussion}

Our observational analysis demonstrates that performing CT scan to diagnose clinically occult pulmonary involvement of COVID-19 in patients who need admission to the hospital for alternative diseases cannot be supported. This is particularly valid in a moment of decrease of the prevalence of the infection during the COVID-19 pandemic.

Our geographic area was first hit by the COVID-19 pandemic outbreak during spring 2020. At that time, our institution identified emergent priorities for the surge of admissions. The priorities included, among others, the need for protecting patients and personnel from the risk of crossinfections inside the hospital. For this reason, it was decided to secure adequate supply of personnel protective equipment and institute separated areas inside the hospital for patients with and without COVID-19 active infection. These areas included beds dedicated both to patients needing intensive or emergency care and to patients needing low-intensity care. Due to the known sub-optimal sensitivity of the first RTPCR testing and delay in obtaining the laboratory result, our group experienced a strategy based on a combination of lung ultrasound and clinical evaluation to guide a safe initial allocation of patients suspected of COVID-19 [8, 9]. The protocol was then validated by an international multicenter study [3]. Based on that experience, we reiterate here that chest imaging needs to be integrated with the clinical condition and presenting symptoms to allow an accurate diagnostic process. After the first allocation in the ED, in cases that remain doubtful, serial repetition of RT-PCR together with extension of chest imaging to CT scan allow completion of the diagnostic process to guide admission of the patients to dedicated COVID or non-COVID areas [3]. During the outbreak waves, the number of alternative pathologies needing hospital admission dramatically fell to a minimum that was never experienced in the past, and almost all the available beds became dedicated to COVID-19 patients. 
Table 1 Correspondence between CT scan readings and clinical diagnoses in 23 patients without symptoms of COVID-19 and negative RT-PCR nasal-pharyngeal swab test, needing hospital admission. The columns report: CT scan results, definitive diagnosis about the sta- tus of SARS-CoV-2 infection during the hospital stay (assigned after follow-up) with main motivation, final clinical diagnosis, dedicated areas inside the hospital where the patients were admitted and then discharged

\begin{tabular}{|c|c|c|c|c|c|}
\hline CT scan reading & COVID-19 status & $\begin{array}{l}\text { Main motivation for COVID-19 } \\
\text { status }\end{array}$ & Final clinical diagnosis & Area of admission & Area of discharge \\
\hline Typical & Negative & Repeated RT-PCR negative & Acute myeloid leukemia & COVID & No COVID \\
\hline Indeterminate & Negative & Follow-up negative & Ischemic stroke & No COVID & No COVID \\
\hline Indeterminate & Negative & COVID-19 positive after months & Subarachnoid hemorrhage & COVID & No COVID \\
\hline Indeterminate & Negative & Pulmonary microcytoma & Obstructive icterus & No COVID & No COVID \\
\hline Indeterminate & Negative & COVID-19 positive after months & Bacterial pneumonia & COVID & No COVID \\
\hline Indeterminate & Negative & Repeated RT-PCR negative & Bacterial pneumonia & COVID & No COVID \\
\hline Indeterminate & Negative & Repeated RT-PCR negative & Bacterial pneumonia & COVID & No COVID \\
\hline Indeterminate & Negative & COVID-19 positive after months & $\begin{array}{l}\text { Third-degree atrio-ventricular } \\
\text { block }\end{array}$ & COVID & COVID \\
\hline Indeterminate & Negative & Repeated RT-PCR negative & $\begin{array}{l}\text { Ischemic stroke bacterial pneu- } \\
\text { monia }\end{array}$ & COVID & No COVID \\
\hline Indeterminate & Negative & Repeated RT-PCR negative & Hepatic failure & No COVID & No COVID \\
\hline Indeterminate & Negative & Repeated RT-PCR negative & Decompensated heart failure & COVID & No COVID \\
\hline Indeterminate & Negative & Repeated RT-PCR negative & Severe COPD in heavy smoker & COVID & No COVID \\
\hline Indeterminate & Negative & Repeated RT-PCR negative & Decompensated heart failure & No COVID & No COVID \\
\hline Indeterminate & Negative & Repeated RT-PCR negative & Bacterial pneumonia & No COVID & No COVID \\
\hline Indeterminate & Negative & COVID-19 positive after months & Decompensated heart failure & COVID & No COVID \\
\hline Indeterminate & Negative & Repeated RT-PCR negative & Bacterial pneumonia & COVID & No COVID \\
\hline Indeterminate & Negative & Repeated RT-PCR negative & Acute myeloid leukemia & No COVID & No COVID \\
\hline Indeterminate & Negative & Repeated RT-PCR negative & Neoplastic pleural effusion & COVID & No COVID \\
\hline Indeterminate & Negative & Pulmonary metastases & Prostate carcinoma & No COVID & No COVID \\
\hline Indeterminate & Negative & Repeated RT-PCR negative & Follicolar lymphoma & COVID & No COVID \\
\hline Indeterminate & Negative & Repeated RT-PCR negative & Septic shock & COVID & Dead \\
\hline Indeterminate & Negative & Repeated RT-PCR negative & Decompensated heart failure & COVID & No COVID \\
\hline Indeterminate & Negative & Repeated RT-PCR negative & Bacterial pneumonia & COVID & No COVID \\
\hline
\end{tabular}

$C T$, computerized tomography of the chest; COVID-19, coronavirus disease 2019; RT-PCR, reverse transcriptase-polymerase chain reaction; SARS-CoV-2, severe acute respiratory syndrome coronavirus 2

However, during these last 2 years, it became evident that the pandemic may manifest with highly variable prevalence [10]. After the first dramatic wave, our institution experienced a fall in the prevalence of COVID-19 and an increase in the number of alternative pathologies needing admissions. Progressively, the COVID-19-dedicated beds were converted to general use, with a growing need to limit the risk of subclinical COVID-19 cases admitted in COVID-19-free areas. To this aim, in our institution, it was deliberated the implementation of a diagnostic algorithm based on the evaluation by CT scan to diagnose clinically occult COVID-19 pneumonia [5]. This algorithm was applied to any patient over 50 years old who needed admission from the ED for any diagnosis alternative to COVID-19 and showing negative RT-PCR tests. The protocol was approved and applied during the period of our analysis; after 3 months of application, our analysis demonstrates its failure.
One of the main objective reasons of failure is the variability in the prevalence of COVID-19. This variability strongly affects the diagnostic accuracy of RT-PCR and chest imaging, the two main diagnostic tools in the management of COVID$19[4,11]$. For example, during the peak of the surge, the probability that a positive RT-PCR assay is effectively linked to the SARS-CoV-2 infection is extremely high and negativity of the test will have not enough power to exclude, while the opposite happens when the prevalence of the infection drops down. Thus, a negative RT-PCR during a fall in the prevalence is far more sensitive in excluding than during the peak of the surge. The same can be said for chest imaging. In a moment of high prevalence, signs of interstitial pneumonia become highly specific, while they fail in ruling-in COVID-19 pneumonia when the prevalence approaches 0 cases and differential diagnoses become more probable. Moreover, chest imaging can only diagnose the pulmonary involvement during the time course of the disease but cannot be of any help in 
diagnosing the infection not complicated by pneumonia. Thus, the usefulness of CT scan when used to diagnose COVID-19 is strongly limited [11, 12]. Finally, following the Bayesian reasoning, the accuracy of any diagnostic test fails when applied to patients without clinical suspicion. Thus, like for any other test, CT scan simply loses specificity in diagnosing COVID19 pneumonia when applied to patients without symptoms of the disease [13].

In the opinion of these authors, a strategy based on systematic RT-PCR and CT imaging for the identification of COVID-19-infected patients in the ED to guide allocation in watertight compartments inside the hospital may hide pitfalls. Based on doubtful imaging obtained during our study, some patients were allocated in COVID-19 areas, even if sometimes in protected beds, but then revealed not infected. Moreover, our observation is limited by the fact that the algorithm was applied in a moment of fall in the prevalence when the probability of false negative RT-PCR became extremely low. It is not said that, during a rise in the prevalence, the percentage of false negative swabs might become significantly higher. In these cases, CT imaging may be of help in finding clinically occult COVID-19 pneumonia but cannot help in diagnosing those cases without pulmonary involvement. For all these reasons, more than relying on a strict selection in the ED of COVID-19 and non-COVID-19 patients for admissions in separated areas, it is safer considering any patient and personnel a possible source of infection. We speculate that a general strategy based on reinforcement of bed distancing, personnel equipping, and strict monitoring of patients with negative RT-PCR tests inside the areas dedicated to alternative COVID-19 pathologies remains a more efficient and safer alternative.

\section{Conclusion}

During the COVID-19 pandemic, the risk of admitting to the hospital patients with asymptomatic SARS-CoV-2 infection should always be considered. However, a systematic CT scan to visualize signs of viral pneumonia cannot be considered a valid strategy to diagnose patients with asymptomatic COVID-19.

\section{Declarations}

Conflict of interest The authors declare that they have no conflict of interest.

\section{References}

1. Li Y, Yao L, Li J, Chen L, Song Y, Cai Z, Yang C (2020) Stability issues of RTPCR testing of SARS-CoV-2 for hospitalized patients clinically diagnosed with COVID-19. J Med Virol 92:903-908
2. Han R, Huang L, Jiang H, Dong J, Peng H, Zhang D (2020) Early clinical and CT manifestations of coronavirus disease 2019 (COVID-19) pneumonia. AJR Am J Roentgenol 215:338-343

3. Volpicelli G, Gargani L, Perlini S, Spinelli S, Barbieri G, Lanotte A, Casasola GG, Nogué-Bou R, Lamorte A, Agricola E, Villén T, Deol PS, Nazerian P, Corradi F, Stefanone V, Fraga DN, Navalesi P, Ferre R, Boero E, Martinelli G, Cristoni L, Perani C, Vetrugno L, McDermott C, Miralles-Aguiar F, Secco G, Zattera C, Salinaro F, Grignaschi A, Boccatonda A, Giostra F, Infante MN, Covella M, Ingallina G, Burkert J, Frumento P, Forfori F, Ghiadoni L; on behalf of the International Multicenter Study Group on LUS in COVID-19(2021) Lung ultrasound for the early diagnosis of COVID-19 pneumonia: an international multicenter study. Intensive Care Med 47:444-454

4. Arevalo-Rodriguez I, Buitrago-Garcia D, Simancas-Racines D, Zambrano-Achig P, Del Campo R, Ciapponi A, Sued O, Martínez-García L, Rutjes A, Low N, Bossuyt PM, Perez-Molina JA, Zamora J (2020) False-negative results of initial RT-PCR assays for COVID-19: a systematic review. https://www.medrx.iv.org/ content/10.1101/2020.04.16.20066787v1

5. Ai T, Yang Z, Hou H, Zhan C, Chen C, Lv W, Tao Q, Sun Z, Xia L (2020) Correlation of chest CT and RT-PCR testing for coronavirus disease 2019 (COVID-19) in China: a report of 1014 cases. Radiology 296:E32-E40

6. Scagliotti G, Novello S, Veltri A, Boccuzzi A, Perboni A, Terzolo M (2020) Patients with lung cancer and coronavirus disease 2019 epidemic: an experience from an Italian university hospital. JTO Clin Res Rep 1:100067. https://doi.org/10.1016/j.jtocrr.2020. 100067

7. Simpson S, Kay FU, Abbara S, Bhalla S, Chung JH, Chung M, Henry TS, Kanne JP, Kligerman S, Ko JP, Litt H (2020) Radiological Society of North America expert consensus statement on reporting chest CT findings related to COVID-19. Endorsed by the Society of Thoracic Radiology, the American College of Radiology, and RSNA. J Thorac Imaging 35:219-227

8. Volpicelli G, Lamorte A, Villén T (2020) What's new in lung ultrasound during the COVID-19 pandemic. Intensive Care Med 46:1445-1448

9. Millington SJ, Koenig S, Mayo P, Volpicelli G (2021) Lung ultrasound for patients with coronavirus disease 2019 pulmonary disease. Chest 159:205-211

10. Aguiar M, Van-Dierdonck JB, Mar J, Cusimano N, Knopoff D, Anam V, Stollenwerk N (2021) Critical fluctuations in epidemic models explain COVID-19 post-lockdown dynamics. Sci Rep 11:13839. https://doi.org/10.1038/s41598-021-93366-7.

11. Hope MD, Raptis CA, Henry TS (2020) Chest computed tomography for detection of coronavirus disease 2019 (COVID-19): don't rush the science. Ann Intern Med 173:147-148

12. Raptis CA, Hammer MM, Short RG, Shah A, Bhalla S, Bierhals AJ, Filev PD, Hope MD, Jeudy J, Kligerman SJ, Henry TS (2020) Chest CT and coronavirus disease (COVID-19): a critical review of the literature to date. AJR Am J Roentgenol 215:839-842

13. Volpicelli G, Cardinale L, Fraccalini T, Calandri M, Piatti C, Geninatti C, Stranieri G (2021) Descriptive analysis of a comparison between lung ultrasound and chest radiography in patients suspected of COVID-19. Ultrasound J 13:11. https://doi.org/10. 1186/s13089-021-00215-9

Publisher's note Springer Nature remains neutral with regard to jurisdictional claims in published maps and institutional affiliations. 